\title{
Intermittency in delay-coupled FitzHugh-Nagumo oscillators and loss of phase synchrony as its precursor
}

\author{
ARINDAM SAHA* and ULRIKE FEUDEL \\ Theoretical Physics/Complex Systems, ICBM, University of Oldenburg, 26129 Oldenburg, Germany \\ *Corresponding author. E-mail: arindam.saha@uni-oldenburg.de
}

\begin{abstract}
We study the dynamical properties of in-out intermittency in a system of two identical FitzHughNagumo oscillators coupled by multiple time delays. In this system, the intermittency is manifested as irregular switching between a nearly synchronous state with small and large amplitude chaotic oscillations and a highly asynchronous state with a single large amplitude oscillation. We show that loss of phase synchrony significantly prior to the occurrence of the asynchronous large amplitude oscillation can be used as a precursor to the switching of states in such systems.
\end{abstract}

Keywords. Delay coupling; on-off intermittency; precursors.

PACS Nos 05.45.-a

\section{Introduction}

Intermittency is a well-known phenomenon in dynamical systems literature and is characterized by an apparently random switching between different types of oscillations [1]. It was first studied by Pomeau and Manneville [2] in maps derived from the Lorenz system. Since then, such intermittency has been observed in a variety of systems including discrete maps [3], coupled oscillators [4], ordinary and partial differential equation models [5], delay-coupled oscillators [6] and stochastic systems [7]. In the deterministic systems, the term intermittency is used in different contexts. On the one hand, it describes one of the major routes to chaos, where three different types of intermittency are distinguished [8]. On the other hand, intermittent dynamics appears in systems containing an invariant manifold. Here the switching of the dynamics between close to the manifold and far from it is described as on-off intermittency [1] or in-out intermittency [5,9]. The study of intermittency has been found to be crucial in understanding many other rich dynamical phenomena including bubbling [10-12] and riddled basins of attraction $[3,13]$.

Based on the exact structure of phase space and the underlying mechanism, intermittency has been classified into categories such as in-out intermittency [5] and on-off intermittency [1]. A feature common to all the classes however is the presence of an invariant (or a quasi-invariant) manifold in the phase space of the system which is repeatedly approached by the trajectory during the dynamics. While such an invariant manifold can be constructed in many other ways, a class of systems which inherently has an invariant manifold is that of coupled identical oscillators. In such a class, the manifold corresponding to complete synchronization of the oscillators is invariant as a result of the exchange symmetry of the systems.

While many studies have focused on the route to intermittency and its dynamical and statistical properties, there are not many works dealing with temporal precursors to the intermittent dynamics. Such an analysis might be crucial as some of the different oscillation regimes among which the dynamical system switches, might be undesirable. A possible example of such a scenario is the study of delay-coupled excitable systems where in-out intermittency has been found as an underlying mechanism of extreme event generation [6].

In addition to intermittency, time-delay systems are known to exhibit interesting dynamical features such as amplitude death [14, 15], multistability [16] and quasiperiodicity [17]. In particular, recent theoretical [18] and experimental [19] studies have indicated various similarities between systems with long delays and spatio-temporal systems. Considerable research interest has also been exhibited in the field of multiple delay systems with novel phenomena such as spiral defects [20] being observed recently. 
In this paper, we consider the same system as in Ref. [6] and focus on a parameter range in which inout intermittency occurs. We introduce a definition of phase such that loss of phase synchrony can be used as a precursor to the intermittent behavior. This system is comprised of two identical FitzHugh-Nagumo (FHN) oscillators which are coupled to each other using multiple time-delay diffusive couplings. Such a form of coupling plays a crucial role in determining the dynamics of various real-life networks where there is more than one connection associated with the same pair of nodes, each corresponding to a different time delay [21-24].

The organization of this paper is as follows. In section 2 , we introduce the system of FHN units under consideration, describe the form of coupling between them and discuss the transverse stability of the invariant synchronization manifold which forms the basis of the intermittency observed. Thereafter, in section 3, we analyze the dynamical regimes where intermittency is observed. Section 4 discusses the phenomenon of intermittency as observed in this system in greater detail. We use a measure to characterize the distance of the trajectory from the invariant manifold; we measure the synchronization error. The dynamics of this synchronization error demonstrates the irregularity of the intermittency and hence motivates the need to identify a precursor. Employing a simple definition of the phase for the oscillators in consideration we show that the phase difference turns out to be a reliable precursor and can hence be used as an early warning signal. Conclusions and future directions of possible research are finally discussed in section 5 .

\section{The model}

We consider a pair of identical FHN units - with internal parameters $a, b$ and $c$-coupled diffusively by multiple delay couplings (see figure 1 ) as follows:

$$
\begin{aligned}
& \dot{x}_{i}=x_{i}\left(a-x_{i}\right)\left(x_{i}-1\right)-y_{i}+\sum_{k=1}^{L} M_{k}\left(x_{j}^{\left(\tau_{k}\right)}-x_{i}\right) \\
& \dot{y}_{i}=b x_{i}-c y_{i}+\sum_{k=1}^{L} M_{k}\left(y_{j}^{\left(\tau_{k}\right)}-y_{i}\right) .
\end{aligned}
$$

Here $i, j \in\{1,2\}, i \neq j$ and

$$
\begin{aligned}
& x_{i}^{\left(\tau_{k}\right)}=x_{i}\left(t-\tau_{k}\right) \\
& y_{i}^{\left(\tau_{k}\right)}=y_{i}\left(t-\tau_{k}\right) .
\end{aligned}
$$

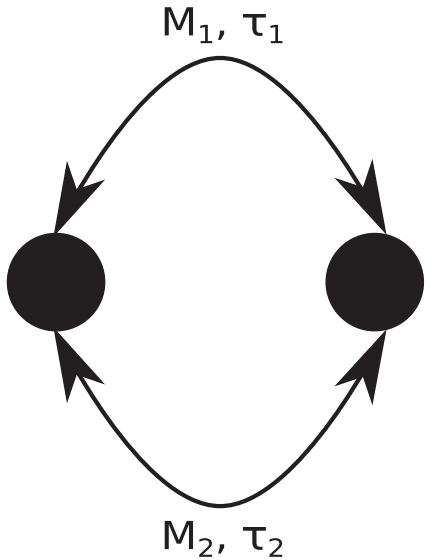

Figure 1. Schematic representation of two FHN units coupled by two delay couplings.

The system is coupled by $L$ delay couplings with each one being characterized by coupling strength $M_{k}$ and time delay $\tau_{k}$. For our study, we use $L=2$ and fix the internal parameters of the independent units at $a=-0.025, b=0.00652$ and $c=0.02$. At these parameter values the units converge to a limit cycle if uncoupled. Furthermore, for the numerical results presented, we assume that for all times $t<0$, the units have the same values as the initial conditions. The results for some other functional forms of histories were also checked and were found to give identical results in the long time limit.

Since the two oscillators are identical and are coupled via a set of diffusive couplings, the completely synchronized state of the system is an invariant set. In other words, if $\tau=\max \left\{\tau_{k}\right\}$ and the trajectory of the system is such that if the $x$ and the $y$ components of the system are identical for at least a time interval of length $\tau$, they will remain identical for all future time. At any time $t$,

$x_{1}\left(t-t^{\prime}\right)=x_{2}\left(t-t^{\prime}\right)$

$y_{1}\left(t-t^{\prime}\right)=y_{2}\left(t-t^{\prime}\right)$

for $0 \leq t^{\prime} \leq \tau$ denotes an invariant manifold of the system. This invariant manifold and its transverse stability play a crucial role in the dynamics of the system as shown in the next section.

\section{Dynamics of the delay-coupled FHN units}

In this section we briefly describe the general qualitative properties of the system described by eq. (1). In the first subsection, we analyze the single-delay system to show the dependence of stability of the invariant manifold 
on the delay of the coupling and examine the emergent dynamics. Thereafter in the second subsection, we use the observations obtained from these single-delay systems to describe the two-delay system where extreme events are observed.

\subsection{One-delay system}

By setting $M_{2}=0$ in eq. (1), we transform the system into a single-delay system with coupling strength $M_{1}$ corresponding to time delay $\tau_{1}$. For the sake of simplicity, we drop the subscripts of the aforementioned parameters and denote them by $M$ and $\tau$ respectively in this subsection. In this scenario, we find that the qualitative dynamics of the system is largely determined by the transverse stability of the synchronization manifold which in turn depends on the time delay $\tau$.

For a large time delay - such as $\tau=80$ - the synchronization manifold is transversally stable. Therefore any trajectory starting away from the manifold converges to it after a short transient behavior. Upon convergence, it executes mixed mode oscillations as illustrated in figure 2 (left column, top row) for $M=0.01$. If $M$ is further increased up to $M=0.014$, the system undergoes a sequence of period-adding bifurcations wherein the FHN units exhibit mixed-mode oscillations inter- spersed by windows of chaotic dynamics. Note that for a transversally stable synchronization manifold, the two FHN units remain in complete synchrony in the long term during both the small amplitude and the large amplitude oscillations (see figure 2 left column, bottom row).

The synchronization manifold loses its transverse stability if the time delay is reduced to $\tau=70$ while keeping the coupling strength fixed at $M=0.01$. In such a scenario, the trajectories starting away from the manifold never converge to it. However, they come very close to the synchronization manifold. This manifests as time intervals of almost complete synchrony of the two FHN units. They then execute some small amplitude oscillations near the synchronization manifold before being ejected away from it (see figure 2 middle column, top row). It is during this time that the FHN units lose their synchrony completely and execute large amplitude oscillations away from the synchronization manifold. After the large amplitude oscillations, the oscillators regain the state of almost complete synchrony as the trajectory in phase space approaches the synchronization manifold and remains in its vicinity to perform small amplitude oscillations (see figure 2 middle column, bottom row).

Note that although the nature of oscillations in the case where the synchronization manifold is transversally unstable is quite distinct from the case when the
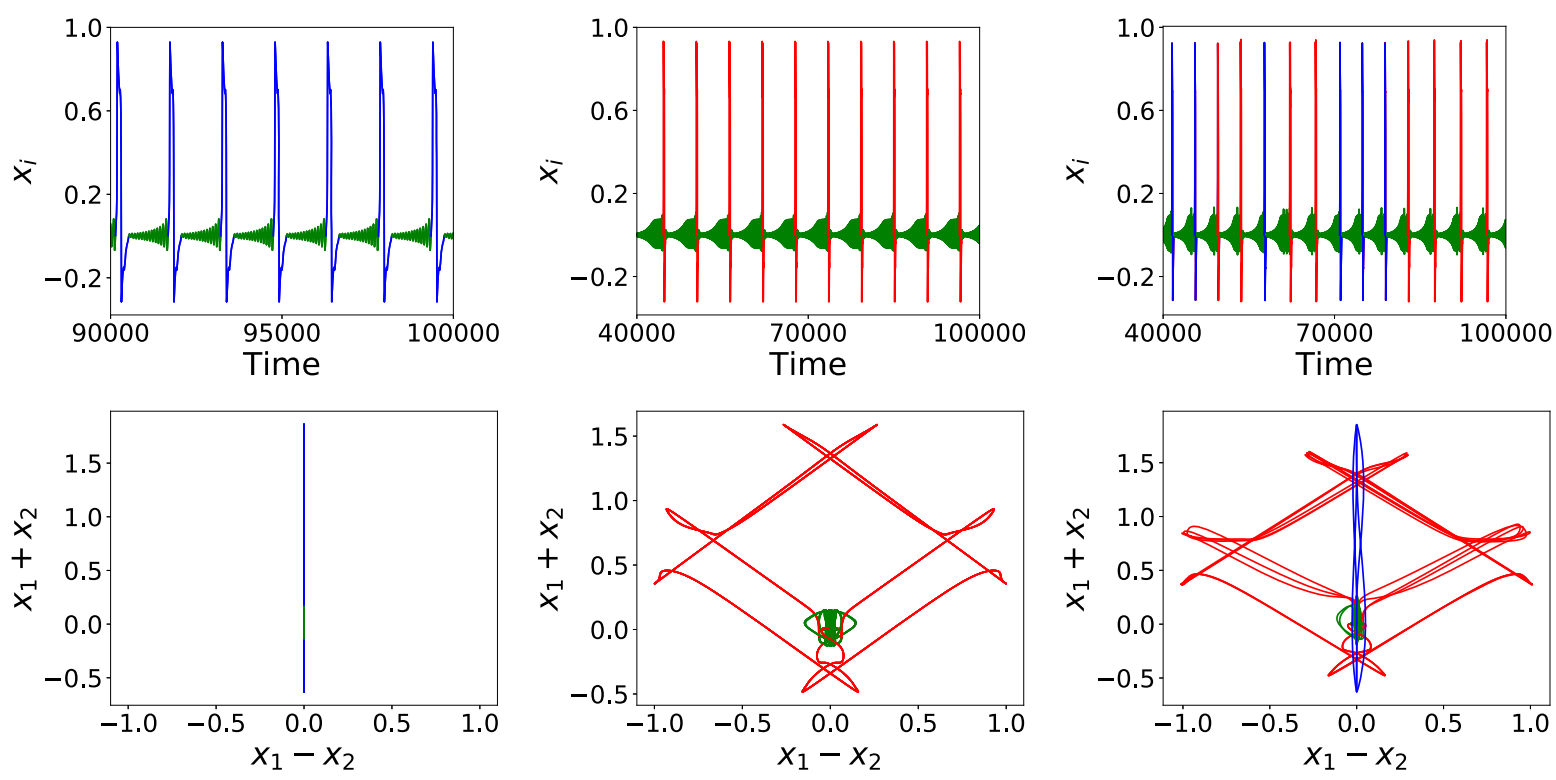

Figure 2. Various representations of the dynamical regimes of the coupled FHN units. Left and middle columns: single-delay coupling with transversally stable and unstable synchronization manifolds respectively. Right column: two-delay coupling. Top row: time evolution of the excitatory dynamical variable. Bottom row: phase space projection with difference and sum of the excitatory variables of both the FHN units plotted on the axes. Color code: small amplitude oscillations in green, synchronous large amplitude oscillations in blue and asynchronous large amplitude oscillations in red. 
synchronization manifold is transversally stable, the two dynamical regimes are similar in the property that the large amplitude oscillations in both cases occur very regularly in time.

In the previous two paragraphs we discussed the qualitative features of the trajectories not starting on the synchronization manifold. Let us now discuss the dynamics of the trajectories which start precisely on the synchronization manifold. Note that since the synchronization manifold is invariant, any trajectory starting on it will never leave it. In this case, we observe that such a trajectory converges to a small amplitude limit cycle on the synchronization manifold. Since this limit cycle is not accessible by any trajectory starting away from the manifold, it may be concluded that it is unstable transversally to the manifold.

Additionally it should be noted that the small amplitude limit cycle is embedded within the nearly synchronous small amplitude oscillations exhibited by the trajectories which do not start on the synchronization manifold. In fact, it is the transverse instability and the position of this limit cycle that causes the asynchronous large amplitude oscillation of the trajectories which do not start on the synchronization manifold. Such trajectories come close to the synchronization manifold due to its attracting nature and execute small amplitude oscillations. During these oscillations they come close to the transversally unstable small amplitude limit cycle and are ejected away from the manifold, finally executing the asynchronous large amplitude oscillation.

If the coupling strength is increased from $M=0.01$ to $M=0.014$ while keeping the time delay fixed at $\tau=70$, the qualitative nature of the dynamics does not change, although a slow gradual decrease in the frequency of the out-of-phase large amplitude oscillations is obtained with increasing in values of $M$.

\subsection{Two-delay system}

In order to study the dynamical properties of the system in the presence of two delay couplings, we set $\tau_{1}=80$, $\tau_{2}=70$ and $M_{1}=0.005$ in eq. (1) and vary $M_{2}$ from 0 to 0.01 . We now briefly describe the dynamical regimes observed as $M_{2}$ is varied.

When $M_{2}=0$, the system effectively has a single delay coupling with $\tau=\tau_{1}=80$. Hence the oscillators get synchronized over time and execute mixed mode oscillations on the synchronization manifold. A gradual increase in $M_{2}$ initially results in a bifurcation sequence identical to the one observed in single-delay systems with a transversally stable synchronization manifold. In particular, until $M_{2} \approx 0.0048$, no asynchronous large amplitude oscillations are observed as the entire long-term motion of the system occurs on the synchronization manifold.

As $M_{2}$ is further increased, a bubbling transition occurs at $M_{2} \approx 0.0048$. After the transition, the synchronization manifold remains transversally stable but the system exhibits very long transients before converging to the chaotic attractor on the manifold. During the transient, the oscillators exhibit almost synchronous small amplitude oscillations interspersed with irregular sequences of almost synchronous and asynchronous large amplitude oscillations (see figure 2 right column) due to an intricate interplay of various invariant sets residing on the synchronization manifold. After this very long transient, the oscillators converge to the chaotic attractor on the synchronization manifold and exhibit synchronized chaotic small amplitude oscillations interspersed with only fully synchronized large amplitude oscillations. Note that the transient times in this case are extremely long in comparison to the transient times in the single-delay case. While the typical transient time of the oscillators coupled using a single delay is typicallly shorter than the time interval between two consecutive large amplitude oscillations, the typical transient time for oscillators coupled by two delays can be as long as several intervals between large amplitude oscillations. The latter occurs particularly when considering the system beyond the bubbling transition.

If the coupling strength is increased beyond $M_{2}$ $\approx 0.0058$, the synchronization manifold loses its transverse stability in a blowout bifurcation. The resulting dynamics is qualitatively identical to that of a singledelay system with a transversally unstable synchronization manifold wherein we observe only asynchronous large amplitude oscillations interspersed with nearly synchronous small amplitude oscillations.

The details of the mechanism by which the abovementioned qualitative changes in dynamics occur can be found in Ref. [6]. There it is also shown that the mechanisms of ejection from and convergence to the synchronization manifold are different. Therefore this type of intermittency is called in-out intermittency according to Ref. [9]. In this paper, we focus only on the parameter region sandwiched between the bubbling transition and the blowout bifurcation where in-out intermittency is observed. In the next section we describe the manifestation of the in-out intermittency in our system in more detail and identify precursors to the formation of asynchronous large amplitude oscillations.

\section{In-Out intermittency and its precursors}

In the previous section, we demonstrated that two identical delay-coupled FHN units can exhibit a dynamical 
state wherein the oscillators execute irregular sequences of asynchronous and synchronous large amplitude oscillations (see figure 3) with long sequences of small amplitude synchronous oscillations separating them. We also discussed that the dynamics is a result of the repeated approach of the trajectory to the invariant synchronization manifold of the system described by eq. (3).

A dynamics in which a trajectory is repeatedly and irregularly ejected away from an invariant manifold of the system, re-approaches it and spends long periods of time in its neighborhood is known as on-off intermittency. In order to characterize this intermittency, we use a distance measure

$d_{\perp}=\sqrt{\left(x_{1}-x_{2}\right)^{2}+\left(y_{1}-y_{2}\right)^{2}}$

such that $d_{\perp}=0$ when the oscillators are on the synchronization manifold; a higher value of $d_{\perp}$ signifies a greater degree of asynchrony. This quantity is also known as synchronization error and measures the deviations of the trajectory from the synchronization manifold.

We track the time evolution of $d_{\perp}$ along a trajectory in the parameter region where the above-mentioned intermittency is observed (see figure 4 middle panel). As expected, $d_{\perp}$ shows peaks only when the asynchronous large amplitude oscillations are observed. During the small amplitude and the synchronous large amplitude oscillations, $d_{\perp}$ remains very close to zero.

If we plot a histogram of time intervals between two consecutive asynchronous large amplitude oscillations (see figure 5), we find that it is exponentially distributed.
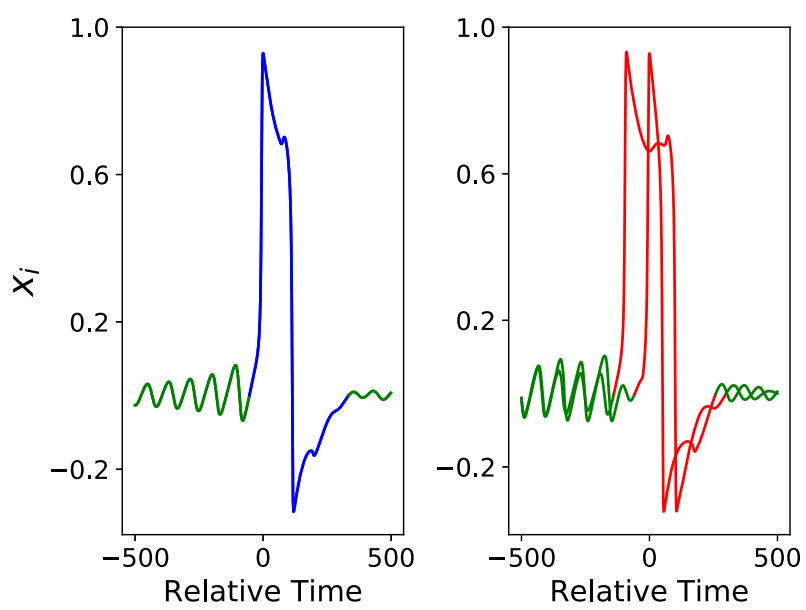

Figure 3. Zoomed-in view of a typical time series showing the $x$ - coordinates of the two coupled FHN unis during a synchronous (left panel) and an asynchronous (right panel) large amplitude oscillation. In both panels, $t=0$ is chosen at the time when the oscillations peak.
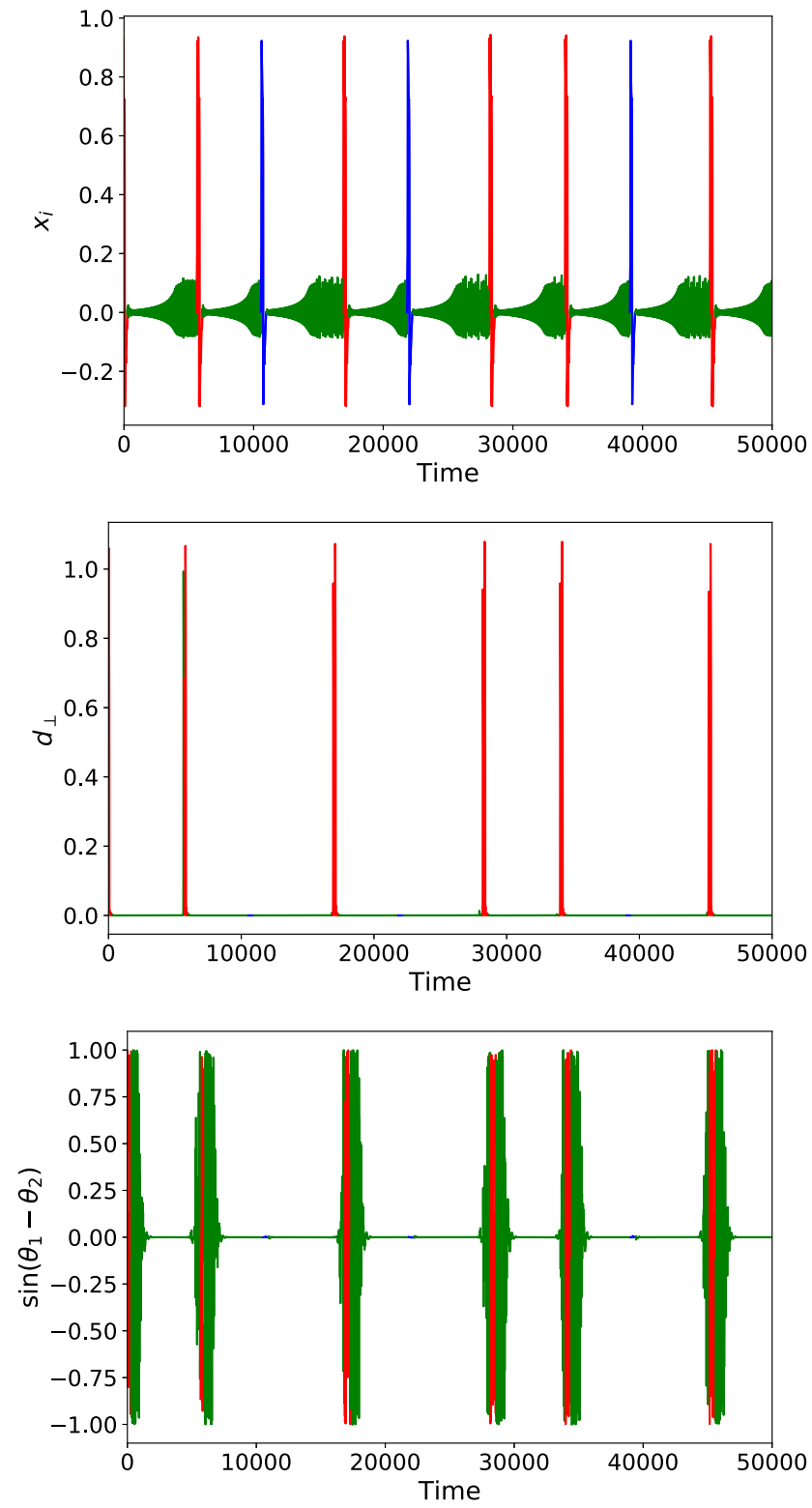

Figure 4. Time evolution of the $x$-coordinates of the twodelay coupled FHN units (top panel) and the distance of the trajectory from the synchronization manifold (middle panel). Bottom panel shows the sine of the difference in phases of the two oscillators in the same time span. Parameters: $M_{1}=0.005, M_{2}=0.0053, \tau_{1}=80$ and $\tau_{2}=70$. Color code: Small amplitude oscillations in green, synchronous large amplitude oscillations in blue and asynchronous large amplitude oscillations in red.

This indicates that this intermittency can be considered as generated from a Poissonian process. It must be noted that due to the chaotic nature of the trajectory, it is not possible to predict the time when the nearly synchronized trajectory is ejected from the invariant manifold to exhibit an asynchronous large amplitude oscillation. Moreover, the time evolution of $d_{\perp}$ does not indicate any 
observable qualitative change prior to an asynchronous large amplitude oscillation.

In the following, our aim is to identify some quantity which could serve as an early warning signal for an upcoming asynchronous large amplitude oscillation. A prerequisite of such a quantity is that during its time evolution it must exhibit two distinct dynamical regimes, one when the trajectory is close to the synchronization manifold, and the other when the trajectory is about to lose synchrony. Moreover, this indicator dynamical variable must transition from the earlier to the later state much before $d_{\perp}$ makes a jump to higher values.

One of the plausible candidates for such a transition is the 'phase difference' between the two FHN units where the phase of each unit is defined as

$\theta_{i}=\tan ^{-1}\left(\frac{y_{i}}{x_{i}}\right)$

with $i \in\{1,2\}$. Note that with this definition, if the trajectory is on the invariant synchronization manifold defined by eq. (3), the phase difference between the two FHN units is zero. In other words, for any trajectory on the synchronization manifold, the FHN units are in phase synchrony. However, a small deviation of the trajectory away from the synchronization manifold need not necessarily translate into a small phase desynchronization. It is this property of this phase definition which can be used to identify precursors to the asynchronous large amplitude oscillations.

If we plot the time evolution of $\sin \left(\theta_{1}-\theta_{2}\right)$ (see figure 4 lower panel), we see that during the synchronous large amplitude oscillations and most of the small amplitude oscillations, the oscillators are phase synchronized. However, prior to the asynchronous large amplitude oscillations, phase synchrony is lost even when the trajectory is still in the neighborhood of the synchronization manifold. This is clearly visible in the close-up view of the time evolution of $\sin \left(\theta_{1}-\theta_{2}\right)$ which peaks prior to the ejection of the trajectory away from the synchronization manifold (see figure 6). The oscillators continue to be out of phase synchrony throughout the large amplitude asynchronous oscillation and even a significant time after the oscillation is complete and the trajectories have returned to the neighborhood of the synchronization manifold.

Since the phase synchrony of the oscillators is lost significantly prior to the asynchronous large amplitude oscillation, loss of phase synchrony can be regarded as a precursor to the ejection of the trajectory away from the synchronization manifold. The length of the time period between the appearance of the precursor

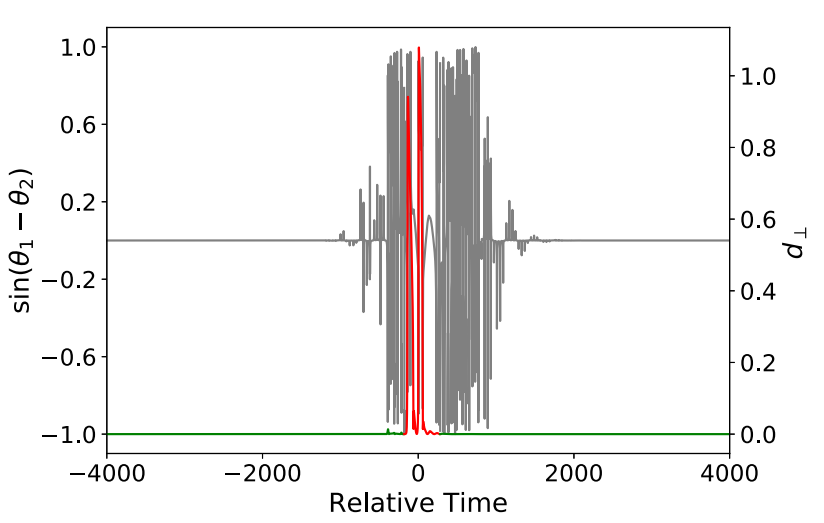

Figure 5. Zoomed-in view of the middle and bottom panels of figure 4 around the time of a asynchronous large amplitude oscillation. The two panels have been plotted on top of each other to highlight the time difference between loss of phase synchrony and the asynchronous large amplitude oscillation. The relative time has been chosen such that the center of the asynchronous large amplitude oscillation occurs at relative time $t=0$.

and the appearance of the asynchronous large amplitude oscillation itself depends on how low we set the threshold defining the loss of synchrony. For example, if we define this threshold as crossing the value 0.01 by $\sin \left(\theta_{1}-\theta_{2}\right)$ for the first time, then the loss in phase synchrony occurs around 488.94 time units prior to the asynchronous large amplitude oscillation. Please note that this time of occurrence of the precursor, here 488.94 time units, is an average over a sample size of 1500 asynchronous large amplitude oscillations. The smaller this threshold, the longer becomes the precursor time. Comparing this to the time required for a typical oscillation - about 80 time units - we see that this

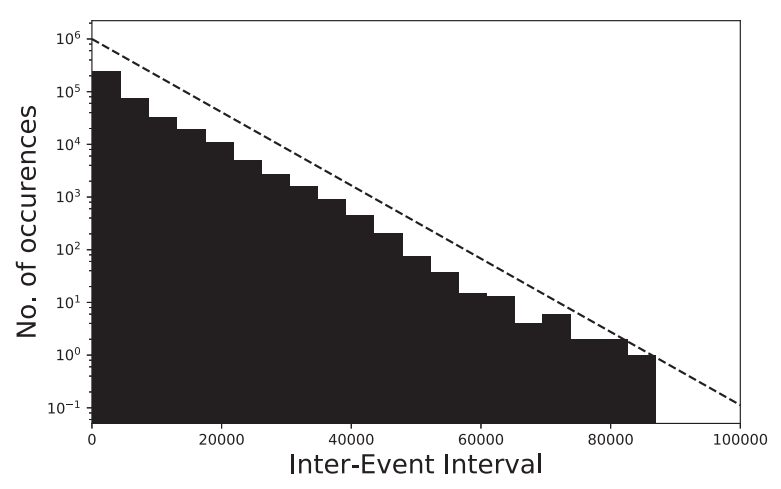

Figure 6. Statistical distribution of inter-event intervals of a trajectory containing 402644 asynchronous large amplitude oscillations. The dashed line represents a fit of $y=\exp (-r t)$ with $r=1.6 \times 10^{-4}$. Parameters: $\tau_{1}=80, \tau_{2}=70$, $M_{1}=0.005$ and $M_{2}=0.0053$. 
precursor appears over 6 oscillations prior to the asynchronous large amplitude oscillation.

\section{Conclusion}

In this study we have presented the loss of phase synchrony as a precursor to in-out intermittency in a pair of identical oscillators coupled by delay diffusive coupling. For the purpose of this analysis, we employ FHN units as the oscillators. Such systems are known to show a highly chaotic dynamics with recurrent large amplitude oscillations interspersed with many small amplitude oscillations. While during all the small amplitude oscillations, the oscillators remain in nearly perfect synchrony, the large amplitude oscillations may occur either synchronously in which the trajectory remains close to the synchronization manifold or asynchronously in which the trajectory is ejected far away from the synchronization manifold during the oscillation. This repeated approach and departure of the trajectory in phase space can be attributed to an in-out intermittency.

Since the ejection events of the trajectory occur irregularly and rarely, it becomes impossible to foresee such an event by direct measurements of the distance of the trajectory from the synchronization manifold. This requires the development of an indirect indicator which can be used as an early warning signal of the forthcoming excursion of the trajectory away from the synchronization manifold. Here we have identified phase synchrony between the oscillators to be such an indicator.

During the small amplitude oscillations and synchronous large amplitude oscillations, the oscillators remain in nearly perfect phase synchrony. However, a few oscillations prior to the ejection of the trajectory away from the synchronization manifold when the trajectory is still close to the synchronization manifold, the phase synchrony between the two FHN units is lost. This loss of phase synchrony continues throughout and beyond the time of ejection of the trajectories. Phase synchrony is regained only few small oscillations after the asynchronous large amplitude oscillation.

Since such a loss in phase synchrony occurs significantly prior to each asynchronous large amplitude oscillation, it can be used as a reliable precursor to such oscillations. The results presented in this paper also indicate that if the phase of oscillators can be appropriately defined, the loss of phase synchrony can be used as a precursor to intermittency in other systems where an invariant manifold is present in the phase space.

\section{Acknowledgements}

The authors wish to thank Gerrit Ansmann, Klaus Lehnertz and Sebastian Wieczorek for valuable discussions. This work was supported by the Volkswagen Foundation (Grants No. 88459). The simulations were performed at the HPC Cluster CARL, located at the University of Oldenburg (Germany) and funded by the DFG through its Major Research Instrumentation Program (INST 184/157-1 FUGG) and the Ministry of Science and Culture (MWK) of the Lower Saxony State.

\section{References}

[1] N Platt, E A Spiegel and C Tresser, Phys. Rev. Lett. 70, 279 (1993)

[2] Y Pomeau and P Manneville, Commun. Math. Phys. 74(2), 189 (1980)

[3] Y-C Lai and C Grebogi, Phys. Rev. E 52, R3313 (1995)

[4] MZhan, GW Wei and C-H Lai, Phys. Rev. E 65, 036202 (2002)

[5] E Covas, R Tavakol, P Ashwin, A Tworkowski and J M Brooke, Chaos 11(2), 404 (2001)

[6] A Saha and U Feudel, Phys. Rev. E 95, 062219 (2017)

[7] T John, R Stannarius and U Behn, Phys. Rev. Lett. 83, 749 (1999)

[8] P Manneville, Dissipative Structures and Weak Turbulence (Perspectives in Physics, Academic Press, 1990)

[9] N Blackbeard, S Wieczorek, H Erzgrber and P S Dutta, Physica D: Nonlinear Phenomena 286-287:43-58 (2014)

[10] V Flunkert, O D'Huys, J Danckaert, I Fischer and E Schöll, Phys. Rev. E 79, 065201 (2009)

[11] S C Venkataramani, B R Hunt, E Ott, D J Gauthier and J C Bienfang, Phys. Rev. Lett. 77, 5361 (1996)

[12] S C Venkataramani, B R Hunt and E Ott, Phys. Rev. E 54, 1346 (1996)

[13] E Ott and J C Sommerer, Phys. Lett. A 188(1), 39 (1994)

[14] K Konishi, K Senda and H Kokame, Phys. Rev. E 78, 056216 (2008)

[15] D Biswas, N Hui and T Banerjee, Nonlinear Dyn. 88(4) (2017)

[16] J Ge, J Xu and Z Li, Nonlinear Dyn. 87(4), 2357 (2017)

[17] M D Shrimali, R Sharan, A Prasad and R Ramaswamy, Phys. Lett. A 374(26), 2636 (2010)

[18] M Wolfrum, S Yanchuk, P Hövel and E Schöll, Eur. Phys. J. Spec. Top. 191(1), 91 (2010)

[19] F Marino, G Giacomelli and S Barland, Phys. Rev. E 95, 052204 (2017)

[20] S Yanchuk and G Giacomelli, Phys. Rev. Lett. 112, 174103 (2014) 
[21] S Bialonski, G Ansmann and H Kantz, Phys. Rev. E 92, 042910 (2015)

[22] S Bialonski, D A Caron, J Schloen, J. Plankton. Res. 38(4), 1077 (2016)
[23] F M Atay and A Hutt, SIAM J. Appl. Math. 65(2), 644 (2004)

[24] F M Atay and A Hutt, J. Appl. Dyn. Syst. 5(4), 670 (2006) 